\title{
Paeoniflorin improves functional recovery through repressing neuroinflammation and facilitating neurogenesis in rat stroke model
}

\author{
Hongli Tang Equal first author, 1 , Leiruo Wu ${ }^{\text {Equal first author, } 2}{ }^{2}$, Xixi Chen ${ }^{1}$, Huiting Li ${ }^{1}$, Baojun Huang ${ }^{1}$, Zhenyang Huang ${ }^{1}$, Yiyang \\ Zheng ${ }^{1}$, Liqing Zhu ${ }^{\text {Corresp., } 3}$, Wujun Geng ${ }^{\text {Corresp. } 1}$ \\ ${ }^{1}$ Anesthesiology, the First Affiliated Hospital of Wenzhou Medical University, Wenzhou, Zhejiang, People's Republic of China \\ 2 \\ 2 Endoscopy center, the First Affiliated Hospital of Wenzhou Medical University, Wenzhou, Zhejiang, People's Republic of China \\ ${ }^{3}$ Clinical Laboratory, the First Affiliated Hospital of Wenzhou Medical University, Wenzhou, Zhejiang, People's Republic of China \\ Corresponding Authors: Liqing Zhu, Wujun Geng \\ Email address: zhuliqing@wzhospital.cn, gengwujun@wzhospital.cn
}

Background. Microglia, neuron, and vascular cells constitute a dynamic functional neurovascular unit, which exerts the crucial role in functional recovery after ischemic stroke. Paeoniflorin, the principal active component of Paeoniae Radix, has been verified to exhibit neuroprotective roles in cerebralischemicinjury. However, the mechanisms underlying the regulatory function of Paeoniflorin on neurovascular unit after cerebral ischemia are still unclear.

Methods. In this study, adult male rats were treated with Paeoniflorin following transient middle cerebral artery occlusion (tMCAO), and then the functional behavioral tests (Foot-fault test and modified improved neurological function score, mNSS), microglial activation, neurogenesis and vasculogenesis were assessed.

Results. The current study showed that Paeoniflorin treatment exhibited a sensorimotor functional recovery as suggested via the Foot-fault test and the enhancement of spatial learning as suggested by the mNSS in rat stroke model. Paeoniflorin treatment repressed microglial cell proliferation and thus resulted in a significant decrease in proinflammatory cytokines IL-1 $\beta$, IL- 6 and TNF- $\alpha$. Compared with control, Paeoniflorin administration facilitated von Willebrand factor (an endothelia cell marker) and doublecortin (a neuroblasts marker) expression, indicating that Paeoniflorin contributed to neurogenesis and vasculogenesis in rat stroke model. Mechanistically, we verified that Paeoniflorin repressed JNK and $\mathrm{NF}-\mathrm{KB}$ signaling activation.

Conclusions. These results demonstrate that Paeoniflorin represses neuroinflammation and facilitates neurogenesis in rat stroke model and might be a potential drug for the therapy of ischemic stroke. 
$1 \quad$ Paeoniflorin improves functional recovery through repressing 2 neuroinflammation and facilitating neurogenesis in rat stroke model

3

4

5

6 1. Anesthesiology, the First Affiliated Hospital of Wenzhou Medical University, Wenzhou, 7 Zhejiang, People's Republic of China.

9 Zhejiang, People's Republic of China.
Hongli Tang ${ }^{1 \#}$, Leiruo $\mathrm{Wu}^{2 \#}$, Xixi Chen ${ }^{1}$, Huiting $\mathrm{Li}^{1}$, Baojun Huang ${ }^{1}$, Zhenyang Huang ${ }^{1}$, Yiyang Zheng ${ }^{1}$, Liqing Zhu ${ }^{3 *}$, Wujun Geng ${ }^{1 *}$
3. Clinical Laboratory, the First Affiliated Hospital of Wenzhou Medical University, Wenzhou, Zhejiang, People's Republic of China.

\#These authors contributed equally: Hongli Tang and Leiruo Wu

*Corresponding authors: Wujun Geng, Department of Anesthesiology, the First Affiliated Hospital of Wenzhou Medical University, Wenzhou, 325000, Zhejiang, People's Republic of China. Tel:86-0577-88581162. Email: gengwujun@wzhospital.cn. Liqing Zhu, Department of Clinical Laboratory, the First Affiliated Hospital of Wenzhou Medical University, Ouhai District, Wenzhou, 325000, Zhejiang, People's Republic of China. Tel:86-0577-55578055. Email: zhuliqing@wzhospital.cn. 


\section{ABSTRACT}

24 Background. Microglia, neuron, and vascular cells constitute a dynamic functional neurovascular unit, which exerts the crucial role in functional recovery after ischemic stroke.

Paeoniflorin, the principal active component of Paeoniae Radix, has been verified to exhibit neuroprotective roles in cerebral ischemic injury. However, the mechanisms underlying the regulatory function of Paeoniflorin on neurovascular unit after cerebral ischemia are still unclear.

Methods. In this study, adult male rats were treated with Paeoniflorin following transient middle cerebral artery occlusion (tMCAO), and then the functional behavioral tests (Foot-fault test and modified improved neurological function score, mNSS), microglial activation, neurogenesis and

32 vasculogenesis were assessed.

33 Results. The current study showed that Paeoniflorin treatment exhibited a sensorimotor

34 functional recovery as suggested via the Foot-fault test and the enhancement of spatial learning

35 as suggested by the mNSS in rat stroke model. Paeoniflorin treatment repressed microglial cell

36 proliferation and thus resulted in a significant decrease in proinflammatory cytokines IL-1 $\beta$, IL-6 
37 and TNF- $\alpha$. Compared with control, Paeoniflorin administration facilitated von Willebrand

38 factor (an endothelia cell marker) and doublecortin (a neuroblasts marker) expression, indicating

39 that Paeoniflorin contributed to neurogenesis and vasculogenesis in rat stroke model.

40 Mechanistically, we verified that Paeoniflorin repressed JNK and NF- $\mathrm{BB}$ signaling activation.

41 Conclusions. These results demonstrate that Paeoniflorin represses neuroinflammation and

42 facilitates neurogenesis in rat stroke model and might be a potential drug for the therapy of 43 ischemic stroke.

44 Keywords: Ischemic stroke, Paeoniflorin, Microglia, Neuroinflammation, JNK.

\section{INTRODUCTION}

47 Ischemic stroke is usually caused by disturbance of the blood supply to the brain, and it posts a

48 significant threat for patients aged over 60 (Hankey 2017; Roy-O'Reilly \& McCullough 2018). It

49 is reported as one of the largest causes of disability and death in the world (Katan \& Luft 2018).

50 Although the incidence, prevalence, and mortality of stroke are decreasing in the US and remain

51 stable worldwide in the past decade (Guzik \& Bushnell 2017), it is still the primary cause of

52 death and mortality in the past two decades in China, especially for patients over 60 years old

53 (Feigin et al. 2014; Moraga \& Collaborators 2017; Wu et al. 2019b; Zhou et al. 2016b). The

54 most common treatments are Intravenous alteplase (rtPA) and endovascular thrombectomy.

55 Intravenous alteplase (rtPA) is the only FDA-approved drug for improving the disability of acute 
56 ischemic stroke patients. It is only effective when given within a very tight time window;

57 otherwise, it may increase the risk of hemorrhagic transformation, and it does not affect mortality

58 (Emberson et al. 2014; Strbian et al. 2014). Endovascular thrombectomy is only used as an

59 additional method for rtPA treatment and it is only effective for $10 \%$ of ischemic stroke patients

60 (Goyal et al. 2016). Furthermore, these treatments have a higher requirement for the local

61 emergency unit on their ability to identify, transfer, and transport them to specialized stroke

62 teams in a short time (Hankey 2017). With the growth of the Chinese population and age, the

63 number of stroke patients will increase significantly and leads to a great financial burden on both

64 the Chinese public health system and families, and it is crucial to develop a new affordable and

65 effective drug for ischemic stroke (Wu et al. 2019b).

67 the pathophysiology of ischemic stroke since it is usually the first immune response step in the

68 central nervous system (Moskowitz et al. 2010). Microglia are soon polarized into either

69 inflammatory phenotype or anti-inflammatory phenotype in the peri-infarct region, based on the

70 stimulus, the period, and the environment (Zong et al. 2019). They can either provoke damage or

71 stimulate neuron repairs according to different pathophysiological conditions (Gordon \& Taylor

72 2005). However, it is reported that microglia activation could cause neurotoxic outcomes by

73 increasing levels of inflammatory cytokines in the long term. (Anttila et al. 2017). Multiple drugs,

74 small molecules, and microRNA are reported in functional recovery of ischemic stroke by

75 adjusting microglia polarization (Ma et al. 2017). 
Paeoniflorin (PF), a monoterpene glucoside purified from the root of Paeonia lactiflora, is

77 reported to have great potential to counter ischemic stroke as an anti-inflammatory and immunosuppressive agent (Xin et al. 2019). According to recent researches, paeoniflorin demonstrate its anti-inflammatory effects in multiple ways: it could reduce the inflammatory cytokine IL-17 and increase the anti-inflammatory cytokine IL-10 (Dai et al. 2015); it also could reduce the response of microglia to injury by suppressing IL-1 $\beta$ and TNF- $\alpha$ level (Zhou et al. 2016a); paeoniflorin also demonstrate its anti-inflammatory ability in multiple pathways by regulating different inflammation-related factors, for example, TNF- $\alpha$, IL- $1 \beta$, TGF- $\beta 1$, Th-1 and etc. (Dai et al. 2015; Jin et al. 2011; Wang et al. 2012; Xin et al. 2019; Zhai \& Guo 2016). These findings not only illustrate the effects of paeoniflorin in multiple inflammatory disorders and neurodegenerative disease but also show the possibility of paeoniflorin using as an antiinflammatory agent targeting at microglia of inflammatory phenotype in ischemic stroke. Some experiments on rats have exhibit paeoniflorin's ability to protect the brain from ischemic damage through inhibiting different inflammatory responses meditated through microglia (Guo et al. 2012a; Tang et al. 2010a; Zhang et al. 2015b). At present, we demonstrated that Paeoniflorin promotes functional recovery by repressing neuroinflammation and facilitating neurogenesis in rat stroke model.

MATERIALS AND METHODS

\section{MCAO model and Paeoniflorin delivery}


96

97

98

99

100

101

102

103

104

105

106

107

108

109

110

111

112

113

114

115

Animal experiments were conduct under the ethic approval obtained from the Committee for Animal Experiments at Wenzhou Medical University (wydw2019-0903). Forty adult male Sprague-Dawley (SD) rats (6-8 weeks, 200-250 g, $\mathrm{n}=10$ of each group) were obtained from the Laboratory Animal Centre Wenzhou Medical University and kept under the specific-pathogenfree (SPF).

MCAO procedures were performed on all groups (the sham-operated group, vehicle experimental group, vehicle Paeoniflorin $5 \mathrm{mg} / \mathrm{kg}$ group and vehicle Paeoniflorin $10 \mathrm{mg} / \mathrm{kg}$ group) as previously described (Hata et al. 1998; Yu et al. 2018). In short, each rat received intraperitoneal anesthesia using choral hydrate $(350 \mathrm{mg} / \mathrm{kg})$ before exposing the correct vessels by a midline skin incision. The middle cerebral artery (MCA) was occluded by inserting a coagulated external carotid artery into the internal carotid artery. The MCA perfusion was allowed by withdrawing the suture after two hours. Verification of the occurrence of ischemia was examined by measuring the blood flow of MCA through a Laser Doppler flowmetry (LDF, Perimed, PeriFlux 5000). The same operations were performed on the sham-operated group rats exclude the MCA occlusion. All rats were kept on a heating pad (Malvern, UK) at $37.0^{\circ} \mathrm{C}$ until the skin incision enclosure. After the previous procedure, the vehicle group was treated with (phosphate-buffered saline consists of $150 \mu$ saline and $20 \%$ dimethyl sulfoxide [DMSO]) by intraperitoneal injection; while the paeoniflorin group was injected with Paeoniflorin $(5 \mathrm{mg} / \mathrm{kg}$ and $10 \mathrm{mg} / \mathrm{kg}$ ) twice per day for 14 days. 
116 light-dark cycle, with food and water available. All rats were sacrificed by overetherization and

117 decapitation.

\section{Behavioral Tests}

119 Neurological function assessment was performed by the mNSS test in accordance with the 120 previous method (Chen et al. 2001). This test was carried out at day 0, 1, 3, 7 and 14 after

121 MCAO. This test, including the measurements for the motor, sensory, beam balance, and 122 reflexes (Schaar et al. 2010). This test was graded on a scale of 0-18. More serious injuries 123 usually lead to higher scores.

125 Zhang (Zhang et al. 2015a). The number of foot-fault errors, paw slipping numbers, were 126 recorded when the rat is trained to cross an elevated grid at day 0,1, 3, 7 and 14 after MCAO.

\section{Cell culture}

128 The murine BV2 microglial cells were procured from the ATCC (Manassas, VA), and then 129 maintained in DMEM with 10\% FBS, 1\% penicillin, and streptomycin (Sigma, MO), and were 130 cultured at $37^{\circ} \mathrm{C}$ in an incubator with $5 \% \mathrm{CO}_{2}$. The oxygen-glucose deprivation (OGD) was 131 performed by exposing BV2 cells to DMEM without glucose or serum and incubating in a 132 specific environment $\left(5 \% \mathrm{CO}_{2}\right.$ and $\left.95 \% \mathrm{~N}_{2}\right)$ for $6 \mathrm{~h}$. BV2 microglial cells were then incubated in 133 Paeoniflorin $(10 \mu \mathrm{M})$ for $6 \mathrm{~h}$ before TNF- $\alpha$, IL-1 $\beta$, IL-6 levels were measured by qPCR analysis. 
135 The total RNA was isolated from BV2 cells or OGD model cells in the absence or presence of

$13610 \mu \mathrm{M}$ Paeoniflorin treatment for $6 \mathrm{~h}$ with Trizol kit (Solarbio, Beijing, China) following the

137 manufacturer's manual. Reverse transcription (RT) was implemented by Prime Script ${ }^{\mathrm{TM}}$ Master $^{-1}$

138 Mix (Katara, Japan). qPCR was performed with $2 \times$ SYBR Green Mix (Solarbio) on an Applied

139 Biosystems 7500 Fast Real-Time PCR Systems (Thermo Fisher Scientific, MA). $\beta$-actin was

140 chosen as an internal reference and the fold changes were calculated using the $2^{-\Delta \Delta \mathrm{Ct}}$ method.

\section{Western blotting}

142 Total protein was purified from the brain tissues of 14 days after MCAO in the absence or 143 presence of $10 \mathrm{mg} / \mathrm{kg}$ Paeoniflorin treatment using lysis buffer and then centrifuged at 12,000 $144 \mathrm{rpm}$ for about $15 \mathrm{~min}$. The protein concentration was measured with the BCA kit (Pierce, IL). 145 The western blotting was carried out as the method reported by Zhu (Zhu et al. 2019). The cell 146 was cultured with primary antibodies, anti-Iba-1 antibody $(1 \mu \mathrm{g} / \mathrm{ml}$; ab5076; Abcam, MA), anti147 JNK antibody (1:1000; ab179461; Abcam, MA), anti-phosphorylated-JNK antibody (1:1,000; 148 \#9251; Cell Signaling Technology), anti-P65 antibody (0.5 $\mu \mathrm{g} / \mathrm{ml} ;$ ab16502; Abcam), anti149 Histone H3 antibody (1:1,000; ab215728; Abcam) and anti-actin antibody (1:1000, ab6276, $150 \mathrm{Abcam}$ ), at $4^{\circ} \mathrm{C}$ overnight. After washing, horseradish peroxidase (HRP)-conjugated secondary 151 antibodies were incubated with membranes for $1 \mathrm{~h}$.

\section{2 (Enzyme linked immunosorbent assay) ELISA}

153 The ELISA assay was carried out to detect TNF- $\alpha$, IL- $1 \beta$ and IL- 6 content using ELISA kits 
154 (PT516/PI303/PI328; Beyotime, China) following the manufacture's manual. In short, the brain

155 tissues were extracted at 14 days after MCAO in the absence or presence of $10 \mathrm{mg} / \mathrm{kg}$

156 Paeoniflorin treatment, and then the supernatant of the brain tissue homogenate (1:20 dilution)

157 was added to 96-well plates that are coated with indicated antibodies, in order to quantify the

158 protein level of TNF- $\alpha$, IL-1 $\beta$, IL-6 in the tissue of the brain. After the reaction was completed,

159 the absorbances of the sample were measured at $450 \mathrm{~nm}$ with a microplate reader. At least three

160 repeated measurements were taken for each sample.

\section{Immunofluorescence staining}

162 The immunofluorescence staining for brain tissues was carried out as the method reported by

163 Burton (Burton et al. 2011), and the brain tissues were extracted at 14 days after MCAO in the

164 absence or presence of $10 \mathrm{mg} / \mathrm{kg}$ Paeoniflorin treatment. The cell was cultured with specific

165 primary antibody against Iba- $1\left(1: 1,500 ;\right.$ ab178846) at $4^{\circ} \mathrm{C}$ overnight, and then incubated with

166 Anti-rat Alexa Fluor $488(1: 1000 ;$ CST) as a secondary antibody. The samples were evaluated

167 with the LEICA TCS SPE microscope (Leica, Germany).

\section{Statistics}

169 Data from the experiments were presented as mean \pm standard deviation (SD).

170 The difference between two groups was compared using two-tailed student's t-test, or one-

171 way analysis of variance (ANOVA) followed by the Scheffé test. Values less than 0.05 was

172 considered significant differences. 


\section{RESULTS}

Paeoniflorin improved the functional recovery in rat stroke model

The role of Paeoniflorin in functional recovery following cerebral ischemic injury was first tested in rat stroke model. The rats were subjected to MCAO and then Paeoniflorin was intraperitoneally administrated for 14 days at $2 \mathrm{~h}$ after MCAO. The Foot-fault test and mNSS were performed at day $0,1,3,7$ and 14 after MCAO. There were no remarkable differences in neurological functional deficits at day 1 after Paeoniflorin administration, while Paeoniflorin markedly improved functional recovery at day 3, 7 and 14 following MCAO, compared with vehicle experimental group (Figure 1A). Meanwhile, Paeoniflorin administration significantly reduced the frequency of forelimb and foot faults at day 3, 7 and 14 following MCAO (Figure 1B). Importantly, better protective effects were obtained with higher Paeoniflorin concentrations in rat stroke model (Figure 1A and B). The current data verified that Paeoniflorin enhanced the functional recovery in rat stroke model.

\section{Paeoniflorin repressed microglia activation in rat stroke model}

Microglia are the resident innate immune cells in the brain. Microglia in resting state are responsible for routine immune surveillance (Guruswamy \& ElAli 2017). After cerebral ischemic injury, microglia are activated in morphological and phenotypical changes (Jayaraj et al. 2019), and the severity of cerebral ischemic injury is correlated with the activated 
192 state of microglia (Emmrich et al. 2015). To explore whether Paeoniflorin repressed the 193 activation of microglia in rat stroke model, microglia were analyzed using immunofluorescence 194 analysis after Paeoniflorin administration. As shown in Figure 2A, rats subjected to MCAO 195 showed a significant microglial proliferation in ipsilateral cortex, whereas additional 196 Paeoniflorin treatment repressed MCAO-induced microglial proliferation. Moreover, 197 Paeoniflorin inhibited MCAO-induced microglial activation as measured by the intensive 198 ramified Iba-1-positive staining (Figure 2A). To further assess the function of Paeoniflorin on microglial activation, the protein expression of Iba-1 in ipsilateral cortex was analyzed. Rats subjected to MCAO showed a significant increase of Iba-1 protein level in ipsilateral cortex, whereas Paeoniflorin treatment inhibited this increase compared with the control (Figure 2B and cytokines was assessed in cerebral tissues. Figure $3 \mathrm{~A}-\mathrm{C}$ showed that rats subjected to MCAO showed a significant increase in the production of IL-1 $\beta$, TNF- $\alpha$ and IL-6, whereas Paeoniflorin administration repressed pro-inflammatory cytokines production in cerebral tissues. These results demonstrated that Paeoniflorin exerts the important role in repressing microglial activation and subsequent neuroinflammation in rat stroke model.

\section{Paeoniflorin repressed microglial viability and inflammatory cytokines production in vitro}

To test the role of Paeoniflorin in microglial activation in vitro, the viability of murine BV-2 
212 Paeoniflorin treatment under normoxia or OGD, indicating that Paeoniflorin inhibited BV-2 cells

213 activity. The effect of Paeoniflorin on pro-inflammatory cytokines production in vitro was

214 further assessed. Here OGD model was used as an in vitro model of ischemia. The mRNA

215 expression level of IL-1 $\beta$, TNF- $\alpha$ and IL-6 was assayed by Real time-PCR under OGD in the

216 presence or absence of Paeoniflorin, and 18s was used as internal gene to control the different

217 amount of RNA input due to the different cell viability. Figure 4B-D showed that IL-1 $\beta$, TNF- $\alpha$

218 and IL-6 expression level was upregulated in BV-2 cells under OGD, but additional treatment of

219 Paeoniflorin repressed OGD-induced increase in IL-1 $\beta$, TNF- $\alpha$ and IL-6 expression. These data

220 suggested that Paeoniflorin improved functional recovery, at least in part, by suppressing

221 microglia-mediated inflammatory response.

\section{Paeoniflorin facilitated neurogenesis and vasculogenesis in rat stroke model}

223 The marker of endothelial cells of cerebral blood vessels, vWF, was next assessed after

224 Paeoniflorin administration. As shown in Figure 5A and B, Paeoniflorin treatment resulted in a

225 significant upregulation of the vWF expression in peri-infarct zone compared with control in rat

226 stroke model. The indication of migrating neuroblasts, doublecortin, was also assessed in peri-

227 infarct zone after Paeoniflorin treatment. Figure 5C and D showed that Paeoniflorin treatment

228 resulted in a significant increase of the doublecortin expression in peri-infarct zone compared

229 with control. Furthermore, Paeoniflorin reduced the TUNEL-positive neurons compared with

230 control (Figure 5E and F). These results demonstrated that Paeoniflorin promoted neurogenesis

231 and vasculogenesis after ischemic stroke.

Peer) reviewing PDF | (2020:07:50844:3:0:NEW 14 Jan 2021) 
233 Given the regulatory role of Paeoniflorin in Jun N-terminal kinase (JNK) signaling in many 234 diseases (Liu et al. 2019; Wu et al. 2019a; Zhang et al. 2017a) and the effect of JNK on 235 regulating inflammatory response (Chen et al. 2018), we next investigated whether Paeoniflorin 236 repressed JNK signaling activation in rat stroke model. The results from western blot analysis 237 showed that the level of p-JNK (phosphorylated JNK) was markedly upregulated in cerebral 238 tissues at $4 \mathrm{~h}$ after MCAO, whereas additional treatment of Paeoniflorin weakened the p-JNK 239 level in cerebral tissues compared with control, suggesting that ischemia-induced activation of 240 JNK signaling was repressed by Paeoniflorin administration (Figure 6A and B).

241 It is well-known that JNK could activate NF- $\kappa \mathrm{B}$ signaling to aggravate the progression of 242 inflammatory response (Pan et al. 2013; Zheng et al. 2020). We thus tested whether 243 neuroinflammation-relieving role of Paeoniflorin was associated with NF- $\kappa$ B signaling activation.

244 Figure 6C and D showed that the nuclear protein level of p65 was markedly upregulated in rat 245 stroke model, whereas additional Paeoniflorin treatment weakened the nuclear p65 level in 246 cerebral tissues compared with control.

247 Immunofluorescence analysis also showed that Paeoniflorin administration partially inhibited

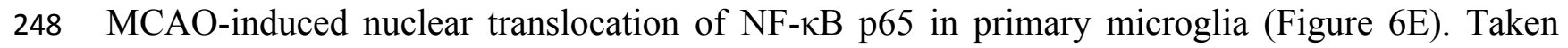
249 together, the current results demonstrated that Paeoniflorin improves functional recovery through 250 repressing neuroinflammation and facilitating neurogenesis by inhibiting JNK- NF- $\kappa$ B signaling 251 in rat stroke model. 


\section{DISCUSSION}

254 In the present study we investigated the potential role of Paeoniflorin in protecting against 255 cerebral ischemia injury, and identified the effect of Paeoniflorin on regulating microglia, neuron, 256 and vascular cells in rat stroke model. The present data verified that (i) Paeoniflorin improved 257 the functional recovery in rat stroke model, (ii) Paeoniflorin repressed microglia activation in rat 258 stroke model, (iii) Paeoniflorin repressed microglial viability and inflammatory cytokines 259 production in vitro, (iv) Paeoniflorin facilitated neurogenesis and vasculogenesis in rat stroke 260 model, (v) Paeoniflorin repressed JNK and NK- $\kappa$ B signaling activation. These data verify the 261 important role of Paeoniflorin in repressing neuroinflammation and facilitating neurogenesis, and 262 may provide a new opportunity to the therapy of ischemic stroke.

263 Paeoniflorin is the primary active component extracted from the roots of Paeonia plants. Over 264 the past decade, the protective role of Paeoniflorin in central nervous system has been verified 265 (She et al. 2019). Paeoniflorin can rapidly pass through the BBB (blood-brain barrier) and 266 repress microglia-mediated inflammatory response in the central nervous system (Liu et al. 267 2006). Our previous study demonstrated that Paeoniflorin administration enhances motor 268 function recovery and reduces the histopathological damage in a rat model of 269 spinal cord injury (Wang et al. 2018). Paeoniflorin increases neuronal survival and represses 270 neuroinflammation by inhibiting NF- $\kappa$ B signaling (Wang et al. 2018). 
demonstrated that Paeoniflorin administration represses MCAO-induced over-activation of microglia and astrocytes, and thus inhibits the production of pro-inflamamtory cytokines including IL-1 $\beta, \mathrm{TNF} \alpha, \mathrm{COX} 2$ and iNOS (Guo et al. 2012b). They further verified that Paeoniflorin exerts a protective role against ischemic injury by inhibiting of JNK, p38 and NFкB signaling activation (Guo et al. 2012b). Ko et al., demonstrated that Paeoniflorin decreases neurological deficit score and enhances motor function (Ko et al. 2018). Tang et al., suggests that both pre-treatment and post-treatment with PF reduced the ratio of cerebral infarction area; pre-treatment with Paeoniflorin also reduced the neurological deficit score (Tang et al. 2010b).

Zhang et al., indicates that the protective effect of Paeoniflorin on cerebral ischemia reperfusion injury is possible through regulating the $\mathrm{Ca} 2+$ CaMKII/CREB signaling pathway(Zhang et al. 2017b). These studies clearly showed the potential role of Paeoniflorin in alleviating ischemic cerebral injury. However, the role and underlying mechanism of paeoniflorin in the neurogenesis

of cerebral ischemia-reperfusion injury are still unclear. Moreover, the current data demonstrated that Paeoniflorin improves functional recovery through repressing neuroinflammation and facilitating neurogenesis in rat stroke model. These data further enhance the persuasiveness of Paeoniflorin in treating ischemic stroke. activated cell types, including microglia and neurons (Huang et al. 2006). Our results document 
291

292

293

294

295

296

297

298

299

300

301

302

303

304

305

306

307

308

309

310

that Paeoniflorin treatment contributes to inhibit microglial cell proliferation and thus results in a significant decrease in proinflammatory cytokines. However, the inhibitory effect of Paeoniflorin treatment on microglia proliferation is due to cytotoxicity or regulation of other signaling pathways remains to be fully elucidated. How does Paeoniflorin regulate the expression of these inflammatory cytokine remains still unclear. Guo et al. demonstrates that Paeoniflorin produces a delayed protection in the ischemia-injured rats via inhibiting MAPKs/NF- $\kappa \mathrm{B}$ mediated peripheral and cerebral inflammatory response(Guo et al. 2012b). We found that ischemia-induced activation of JNK signaling was repressed by Paeoniflorin administration. It is well-known that JNK could activate NF- $\kappa$ B signaling to aggravate the progression of inflammatory response (Pan et al. 2013; Zheng et al. 2020). These data suggest that Paeoniflorin regulate these inflammatory cytokine by suppressing JNK- NF- $\mathrm{B}$ signaling pathway in rat stroke model.

endothelia cell marker) and doublecortin (a neuroblasts marker) expression compared with control, suggesting that Paeoniflorin facilitates neurogenesis and vasculogenesis in rat stroke model. the underlying mechanism of Paeoniflorin facilitates neurogenesis still unclear. It has been reported that Microglia and proinflammatory cytokines can enhance neuroprotection or neurotoxicity during the inflammatory response, so they are a double-edged sword in neurogenesis(Ekdahl et al. 2009). Ekdahl et al., reported that the number of activated microglia was negatively correlated with the survival rate of neonatal hippocampal neurons(Ekdahl et al. 2009). Bangrong Cai et al., Arg-DG attenuates LPS-impaired neurogenesis and suppresses the 
311 production of LPS-induced pro-inflammatory cytokines in hippocampal DG by blocking

312 microglial activation(Cai et al. 2019). Our data suggests Paeoniflorin treatment contributes to

313 inhibit microglial cell proliferation and thus results in a significant decrease in proinflammatory

314 cytokines. Based on these data. we concluded that Paeoniflorin inhibits neuroinflammation and

315 facilitates neurogenesis by suppressing the production of inflammatory cytokine and activation

316 of microglia.

317 In conclusion, we showed that Paeoniflorin exhibits a sensorimotor functional recovery.

318 Paeoniflorin treatment contributes to inhibit microglial cell proliferation and thus results in a

319 significant decrease in proinflammatory cytokines. Meanwhile, Furthermore, we demonstrated

320 that Paeoniflorin represses $\mathrm{JNK}$ and $\mathrm{NF}-\kappa \mathrm{B}$ signaling activation. The current data verify that

321 Paeoniflorin inhibits neuroinflammation and facilitates neurogenesis in rat stroke model and

322 might be a potential drug for the therapy of ischemic stroke.

\section{ACKNOWLEDGMENTS}

\section{Funding}

326 The project was supported by Zhejiang public welfare technology research plan

327 (LGD20H290002), the National Natural Science Foundation of China (No. 81973620,

328 No.81774109), and Major scientific and technological innovation medical and health projects of

329 Wenzhou science and Technology Bureau (ZY2019015). 
331 Conflicts of interest

332 The authors declare that they have no competing of interests.

\section{Author Contributions}

- Hongli Tang, Leiruo Wu and Xixi Chen conceived and designed the experiments.

- Huiting Li, Baojun Huang and Zhenyang Huang performed the experiments.

- Yiyang Zheng, Liqing Zhu and Wujun Geng analyzed the data.

- Hongli Tang, Leiruo Wu, Xixi Chen, Huiting Li and Baojun Huang prepared the figures and/or tables.

- Zhenyang Huang, Yiyang Zheng, Liqing Zhu and Wujun Geng drafted the work or revised it critically for important content.

\section{Data Availability}

343 The following information was supplied regarding data availability: The raw data has been 344 uploaded as a Supplemental File. 
346

347

348

349

350

351

352

353

354

355

356

357

358

359

360

361

362

363

364

365

366

367

368

369

370

371

372

373

\section{References}

Anttila JE, Whitaker KW, Wires ES, Harvey BK, and Airavaara M. 2017. Role of microglia in ischemic focal stroke and recovery: focus on Toll-like receptors. Prog Neuropsychopharmacol Biol Psychiatry 79:3-14.

Burton MD, Sparkman NL, and Johnson RW. 2011. Inhibition of interleukin-6 trans-signaling in the brain facilitates recovery from lipopolysaccharide-induced sickness behavior. Journal of neuroinflammation 8:54.

Cai B, Seong KJ, Bae SW, Kook MS, Chun C, Lee JH, Choi WS, Jung JY, and Kim WJ. 2019. Water-Soluble ArginylDiosgenin Analog Attenuates Hippocampal Neurogenesis Impairment Through Blocking Microglial Activation Underlying NF-kappaB and JNK MAPK Signaling in Adult Mice Challenged by LPS. Mol Neurobiol 56:6218-6238.

Chen J, Sanberg PR, Li Y, Wang L, Lu M, Willing AE, Sanchez-Ramos J, and Chopp M. 2001. Intravenous administration of human umbilical cord blood reduces behavioral deficits after stroke in rats. Stroke $32: 2682-2688$.

Chen X, Li X, Zhang W, He J, Xu B, Lei B, Wang Z, Cates C, Rousselle T, and Li J. 2018. Activation of AMPK inhibits inflammatory response during hypoxia and reoxygenation through modulating JNK-mediated NF-kappaB pathway. Metabolism 83:256-270.

Dai X, Wang L-W, Jia X-Y, Chang Y, Wu H-X, Wang C, and Wei W. 2015. Paeoniflorin regulates the function of human peripheral blood mononuclear cells stimulated by rhlL-1 $\beta$ by up-regulating Treg expression. Immunopharmacology and immunotoxicology 37:252-257.

Ekdahl CT, Kokaia Z, and Lindvall O. 2009. Brain inflammation and adult neurogenesis: the dual role of microglia. Neuroscience 158:1021-1029.

Emberson J, Lees KR, Lyden P, Blackwell L, Albers G, Bluhmki E, Brott T, Cohen G, Davis S, and Donnan G. 2014. Effect of treatment delay, age, and stroke severity on the effects of intravenous thrombolysis with alteplase for acute ischaemic stroke: a meta-analysis of individual patient data from randomised trials. The Lancet 384:1929-1935.

Emmrich JV, Ejaz S, Neher JJ, Williamson DJ, and Baron JC. 2015. Regional distribution of selective neuronal loss and microglial activation across the MCA territory after transient focal ischemia: quantitative versus semiquantitative systematic immunohistochemical assessment. J Cereb Blood Flow Metab 35:20-27.

Feigin VL, Forouzanfar MH, Krishnamurthi R, Mensah GA, Connor M, Bennett DA, Moran AE, Sacco RL, Anderson L, and Truelsen T. 2014. Global and regional burden of stroke during 1990-2010: findings from the Global 
Gordon S, and Taylor PR. 2005. Monocyte and macrophage heterogeneity. Nat Rev Immunol 5:953-964.

Goyal M, Yu AY, Menon BK, Dippel DW, Hacke W, Davis SM, Fisher M, Yavagal DR, Turjman F, and Ross J. 2016. Endovascular therapy in acute ischemic stroke: challenges and transition from trials to bedside. Stroke 47:548-553.

Guo R-B, Wang G-F, Zhao A-P, Gu J, Sun X-L, and Hu G. 2012a. Paeoniflorin protects against ischemia-induced brain damages in rats via inhibiting MAPKs/NF-KB-mediated inflammatory responses. PLoS One 7:e49701.

Guo RB, Wang GF, Zhao AP, Gu J, Sun XL, and Hu G. 2012b. Paeoniflorin protects against ischemia-induced brain damages in rats via inhibiting MAPKs/NF-kappaB-mediated inflammatory responses. PLoS One 7:e49701.

Guruswamy R, and ElAli A. 2017. Complex Roles of Microglial Cells in Ischemic Stroke Pathobiology: New Insights and Future Directions. Int J Mol Sci 18.

Guzik A, and Bushnell C. 2017. Stroke Epidemiology and Risk Factor Management. Continuum (Minneap Minn) 23:15-39.

Hankey GJ. 2017. Stroke. The Lancet 389:641-654.

Hata R, Mies G, Wiessner C, Fritze K, Hesselbarth D, Brinker G, and Hossmann K-A. 1998. A reproducible model of middle cerebral artery occlusion in mice: hemodynamic, biochemical, and magnetic resonance imaging. Journal of Cerebral Blood Flow \& Metabolism 18:367-375.

Huang J, Upadhyay UM, and Tamargo RJ. 2006. Inflammation in stroke and focal cerebral ischemia. Surg Neurol 66:232-245.

Jayaraj RL, Azimullah S, Beiram R, Jalal FY, and Rosenberg GA. 2019. Neuroinflammation: friend and foe for ischemic stroke. J Neuroinflammation 16:142.

Jin L, Zhang LM, Xie KQ, Ye Y, and Feng L. 2011. Paeoniflorin suppresses the expression of intercellular adhesion molecule - 1 (ICAM - 1) in endotoxin - treated human monocytic cells. British journal of pharmacology 164:694-703.

Katan M, and Luft A. 2018. Global Burden of Stroke. Semin Neurol 38:208-211.

Ko $\mathrm{CH}$, Huang CP, Lin YW, and Hsieh CL. 2018. Paeoniflorin has anti-inflammation and neurogenesis functions through nicotinic acetylcholine receptors in cerebral ischemia-reperfusion injury rats. Iran J Basic Med Sci 21:1174-1178.

Peer) reviewing PDF | (2020:07:50844:3:0:NEW 14 Jan 2021) 
403

404

405

406

407

408

409

410

411

412

413

414

415

416

417

418

419

420

421

422

Liang J, Wang J, Saad Y, Warble L, Becerra E, and Kolattukudy PE. 2011. Participation of MCP-induced protein 1 in lipopolysaccharide preconditioning-induced ischemic stroke tolerance by regulating the expression of proinflammatory cytokines. J Neuroinflammation 8:182.

Liu J, Jin DZ, Xiao L, and Zhu XZ. 2006. Paeoniflorin attenuates chronic cerebral hypoperfusion-induced learning dysfunction and brain damage in rats. Brain Res 1089:162-170.

Liu Y, Han J, Zhou Z, and Li D. 2019. Paeoniflorin protects pancreatic beta cells from STZ-induced damage through inhibition of the p38 MAPK and JNK signaling pathways. Eur J Pharmacol 853:18-24.

Ma Y, Wang J, Wang Y, and Yang GY. 2017. The biphasic function of microglia in ischemic stroke. Prog Neurobiol 157:247-272.

Moraga P, and Collaborators GCoD. 2017. Global, regional, and national age-sex specific mortality for 264 causes of death, 1980-2016: a systematic analysis for the Global Burden of Disease Study 2016. The Lancet 390:1151-1210.

Moskowitz MA, Lo EH, and ladecola C. 2010. The science of stroke: mechanisms in search of treatments. Neuron 67:181-198.

Pan Y, Zhang X, Wang Y, Cai L, Ren L, Tang L, Wang J, Zhao Y, Liu Q, Li X, and Liang G. 2013. Targeting JNK by a new curcumin analog to inhibit NF-kB-mediated expression of cell adhesion molecules attenuates renal macrophage infiltration and injury in diabetic mice. PLoS One 8:e79084.

Roy-O'Reilly M, and McCullough LD. 2018. Age and Sex Are Critical Factors in Ischemic Stroke Pathology. Endocrinology 159:3120-3131.

Schaar KL, Brenneman MM, and Savitz SI. 2010. Functional assessments in the rodent stroke model. Exp TransI Stroke Med 2:13.

She Y, Shao L, Zhang Y, Hao Y, Cai Y, Cheng Z, Deng C, and Liu X. 2019. Neuroprotective effect of glycosides in Buyang Huanwu Decoction on pyroptosis following cerebral ischemia-reperfusion injury in rats. $J$ Ethnopharmacol 242:112051.

Strbian D, Michel P, Seiffge DJ, Saver JL, Numminen H, Meretoja A, Murao K, Weder B, Forss N, and Parkkila A-K. 2014. Symptomatic intracranial hemorrhage after stroke thrombolysis: comparison of prediction scores. Stroke 45:752-758.

Tang N-Y, Liu C-H, Hsieh C-T, and Hsieh C-L. 2010a. The anti-inflammatory effect of paeoniflorin on cerebral infarction induced by ischemia-reperfusion injury in Sprague-Dawley rats. The American journal of Chinese medicine 38:51-64.

Peer) reviewing PDF | (2020:07:50844:3:0:NEW 14 Jan 2021) 
433

434

435

436

437

438

439

440

441

442

443

444

445

446

447

448

449

450

451

452

453

454

455

456

457

458

459

460

461

462

Tang NY, Liu CH, Hsieh CT, and Hsieh CL. 2010b. The anti-inflammatory effect of paeoniflorin on cerebral infarction induced by ischemia-reperfusion injury in Sprague-Dawley rats. Am J Chin Med 38:51-64.

Wang B, Dai W, Shi L, Teng H, Li X, Wang J, and Geng W. 2018. Neuroprotection by Paeoniflorin against Nuclear Factor Kappa B-Induced Neuroinflammation on Spinal Cord Injury. Biomed Res Int 2018:9865403.

Wang D, Yuan F, Wang L, and Wei W. 2012. Paeoniflorin inhibits function and down-regulates HLA-DR and CD80 expression of human peripheral blood monocytes stimulated by rhlL-1ß. International immunopharmacology 14:172-178.

Wu F, Ye B, Wu X, Lin X, Li Y, Wu Y, and Tong L. 2019a. Paeoniflorin on Rat Myocardial Ischemia Reperfusion Injury of Protection and Mechanism Research. Pharmacology:1-8.

Wu S, Wu B, Liu M, Chen Z, Wang W, Anderson CS, Sandercock P, Wang Y, Huang Y, Cui L, Pu C, Jia J, Zhang T, Liu X, Zhang S, Xie P, Fan D, Ji X, Wong K-SL, Wang L, Wu S, Wu B, Liu M, Chen Z, Wang W, Anderson CS, Sandercock P, Wang Y, Huang Y, Cui L, Pu C, Jia J, Zhang T, Liu X, Zhang S, Xie P, Fan D, Ji X, Wong K-SL, Wang L, Wei C, Wang Y, Cheng Y, Liu Y, Li X, Dong Q, Zeng J, Peng B, Xu Y, Yang Y, Wang Y, Zhao G, Wang W, Xu Y, Yang Q, He Z, Wang S, You C, Gao Y, Zhou D, He L, Li Z, Yang J, Lei C, Zhao Y, Liu J, Zhang S, Tao W, Hao Z, Wang D, and Zhang S. 2019b. Stroke in China: advances and challenges in epidemiology, prevention, and management. The Lancet Neurology 18:394-405.

Xin Q, Yuan R, Shi W, Zhu Z, Wang Y, and Cong W. 2019. A review for the anti-inflammatory effects of paeoniflorin in inflammatory disorders. Life sciences:116925.

Yu J, Li X, Matei N, McBride D, Tang J, Yan M, and Zhang JH. 2018. Ezetimibe, a NPC1L1 inhibitor, attenuates neuronal apoptosis through AMPK dependent autophagy activation after MCAO in rats. Experimental neurology 307:12-23.

Zhai J, and Guo Y. 2016. Paeoniflorin attenuates cardiac dysfunction in endotoxemic mice via the inhibition of nuclear factor-кB. Biomedicine \& Pharmacotherapy 80:200-206.

Zhang J, Wang F, Wang H, Wang Y, Wu Y, Xu H, and Su C. 2017a. Paeoniflorin inhibits proliferation of endometrial cancer cells via activating MAPK and NF-kappaB signaling pathways. Exp Ther Med 14:5445-5451.

Zhang Y, Chopp M, Meng Y, Katakowski M, Xin H, Mahmood A, and Xiong Y. 2015a. Effect of exosomes derived from multipluripotent mesenchymal stromal cells on functional recovery and neurovascular plasticity in rats after traumatic brain injury. Journal of neurosurgery 122:856-867.

Zhang Y, Li H, Huang M, Huang M, Chu K, Xu W, Zhang S, Que J, and Chen L. 2015b. Paeoniflorin, a monoterpene glycoside, protects the brain from cerebral ischemic injury via inhibition of apoptosis. The American

Peer] reviewing PDF | (2020:07:50844:3:0:NEW 14 Jan 2021) 
journal of Chinese medicine 43:543-557.

Zhang Y, Qiao L, Xu W, Wang X, Li H, Xu W, Chu K, and Lin Y. 2017b. Paeoniflorin Attenuates Cerebral Ischemia-

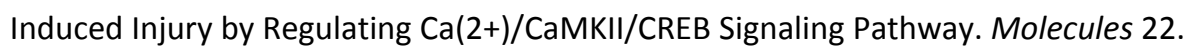

Zheng J, Dai Q, Han K, Hong W, Jia D, Mo Y, Lv Y, Tang H, Fu H, and Geng W. 2020. JNK-IN-8, a c-Jun N-terminal kinase inhibitor, improves functional recovery through suppressing neuroinflammation in ischemic stroke. J Cell Physiol 235:2792-2799.

Zhou J, Wang L, Wang J, Wang C, Yang Z, Wang C, Zhu Y, and Zhang J. 2016a. Paeoniflorin and albiflorin attenuate neuropathic pain via mapk pathway in chronic constriction injury rats. Evidence-Based Complementary and Alternative Medicine 2016.

Zhou M, Wang H, Zhu J, Chen W, Wang L, Liu S, Li Y, Wang L, Liu Y, and Yin P. 2016b. Cause-specific mortality for 240 causes in China during 1990-2013: a systematic subnational analysis for the Global Burden of Disease Study 2013. The Lancet 387:251-272.

Zhu Y, Wu Y, Yang L, Dou X, Jiang J, and Wang L. 2019. Long non - coding RNA activated by transforming growth factor - $\beta$ promotes proliferation and invasion of cervical cancer cells by regulating the miR - 144/ITGA6 axis. Experimental physiology.

Zong X, Dong Y, Li Y, Yang L, Li Y, Yang B, Tucker L, Zhao N, Brann DW, Yan X, Hu S, and Zhang Q. 2019. Beneficial Effects of Theta-Burst Transcranial Magnetic Stimulation on Stroke Injury via Improving Neuronal Microenvironment and Mitochondrial Integrity. Trans/ Stroke Res.

\section{Figure legend}

\section{Figure 1. Paeoniflorin improved the functional recovery in rat stroke model}

(A) The neurological function of rats was measured by mNS at day 0, 1, 3, 7 and 14 after MCAO and intraperitoneal injection of vehicle or Paeoniflorin $(5 \mathrm{mg} / \mathrm{kg}$ and $10 \mathrm{mg} / \mathrm{kg}$; $\mathrm{n}=10$ of each group). Data was analyzed by one-way ANOVA followed by the Scheffé test. $\mathrm{n}=10$. $* * P<0.01$ vs. control group. (B) A foot-fault test was performed at day $0,1,3,7$ and 14 after MCAO and intraperitoneal injection of vehicle or Paeoniflorin $(5 \mathrm{mg} / \mathrm{kg}$ and $10 \mathrm{mg} / \mathrm{kg})$. Data was 
488

489

490

491

492

493

494

495

496

497

498

499

500

501

502

503

504

505

506

analyzed by one-way ANOVA followed by the Scheffé test. $\mathrm{n}=10{ }^{* *} P<0.01$ vs. control group. mNSS, Modified neurological severity scores; MCAO, middle cerebral artery occlusion.

\section{Figure 2. Paeoniflorin repressed microglia activation in rat stroke model}

(A) The activation of microglia was analyzed using immunofluorescence analysis in ipsilateral cortex after MCAO and intraperitoneal injection of vehicle or Paeoniflorin $(10 \mathrm{mg} / \mathrm{kg}) \mathrm{n}=3$. Scale bar, $20 \mu \mathrm{m}(\mathrm{B}$ and $\mathrm{C}$ ) Western blot and quantitative analysis of the protein expression level of Iba-1 in ipsilateral cortex after MCAO and intraperitoneal injection of vehicle or Paeoniflorin. $* P<0.05, * * P<0.01 . \mathrm{n}=3$. MCAO, middle cerebral artery occlusion; Iba-1, Ionized calcium bindingadaptor molecule-1.

Figure 3. Paeoniflorin inhibits the secretion of pro-inflammatory cytokines in rat stroke model

(A-C) The levels of pro-inflammatory cytokines TNF-a (A), IL-1 $\beta$ (B) and IL-6 (C) were determined by ELISA assay using ELISA kits in brain tissues after MCAO and intraperitoneal injection of vehicle or Paeoniflorin $(10 \mathrm{mg} / \mathrm{kg}) . \mathrm{n}=3 . * P<0.05, * * P<0.01$. IL-1 $\beta$, Interleukin-1 beta; TNF, tumor necrosis factor; IL-6, Interleukin-6; ELISA, enzyme linked immunosorbent assay; MCAO, middle cerebral artery occlusion.

Figure 4. Paeoniflorin repressed microglial viability and inflammatory cytokines production in vitro 
507 vehicle or Paeoniflorin treatment $(10 \mu \mathrm{M})$ and OGD models (BV2 cells subjected to oxygen and

508 glucose deprivation) treated with vehicle or Paeoniflorin treatment $(10 \mu \mathrm{M})$ for $24 \mathrm{~h}$. $\mathrm{n}=3$.

$509 * P<0.05$. (B-D) The mRNA expression levels of IL-1 $\beta$ (B), TNF- $\alpha$ (C), and IL-6 (D) were

510 detected by qPCR analysis in BV2 cells with vehicle or Paeoniflorin treatment $(10 \mu \mathrm{M})$ and OGD

511 models treated with vehicle or Paeoniflorin treatment $(10 \mu \mathrm{M})$ for $24 \mathrm{~h} . \mathrm{n}=3 . * * P<0.01$. CCK-8,

512 Cell Counting Kit-8; OGD, oxygen-glucose deprivation; IL-1 $\beta$, Interleukin-1 beta; TNF, tumor

513 necrosis factor; IL-6, Interleukin-6; qPCR, Quantitative Real-time PCR.

514 Figure 5. Paeoniflorin facilitated neurogenesis and vasculogenesis in rat stroke model

515 (A and B) The marker of endothelial cells of cerebral blood vessels (vWF) expression was

516 assessed in peri-infarct zone compared with control of rat stroke model after Paeoniflorin

517 administration by Immunofluorescence. $\mathrm{n}=3$. $* P<0.05$. ( $\mathrm{C}$ and $\mathrm{D})$ The indication of migrating

518 neuroblasts (doublecortin) expression was assessed in peri-infarct zone compared with control of

519 rat stroke model after Paeoniflorin administration by Immunofluorescence. $* * P<0.01$. (E and F)

520 The effects of paeoniflorin on apoptotic cell was verified by TUNEL staining in rat stroke model

521 treated with or without Paeoniflorin. Positive expression in TUNEL staining was exhibited. Rats

522 treated with saline and PF $(10 \mathrm{mg} / \mathrm{kg})$ for 14 days after MCAO; $\mathrm{n}=5$. Scale bar, $50 \mu \mathrm{m}$.

$523 * * P<0.01$. TUNEL, terminal dexynucleotidyl transferase (TdT)-mediated dUTP nick end

524 labeling.

525 Figure 6. Paeoniflorin repressed JNK and NK- $\kappa B$ signaling activation 
526 (A and B) Western blot and quantitative analysis of the protein expression level of JNK and 527 phosphorylated JNK (p-JNK) after MCAO and intraperitoneal injection of vehicle or 528 Paeoniflorin. $\mathrm{n}=3$. $* * P<0.01, \# P<0.05$. (C and D) Western blot and quantitative analysis of the 529 protein expression level of nuclear p65 and Histone H3 after MCAO and intraperitoneal injection 530 of vehicle or Paeoniflorin. (E) Nuclear translocation of p65 was indicated using an 531 immunofluorescence assay after MCAO and intraperitoneal injection of vehicle or Paeoniflorin. $532 \mathrm{n}=3 .{ }^{*} P<0.05$. Scale bar, $20 \mu \mathrm{m}$. MCAO, middle cerebral artery occlusion. 


\section{Figure 1}

Paeoniflorin improved the functional recovery in rat stroke model

(A) Theneurological function of rats was measured by mNSS at day 0, 1, 3, 7 and 14 after MCAO and intraperitoneal injection of vehicle or Paeoniflorin $(5 \mathrm{mg} / \mathrm{kg}$ and $10 \mathrm{mg} / \mathrm{kg} ; \mathrm{n}=10$ of each group). Data was analyzed by one-way ANOVA followedbytheScheffétest. $n=10$. $* * P<0.01$ vs. control group. (B) A foot-fault test was performed at day $0,1,3,7$ and 14 after MCAO and intraperitoneal injection of vehicle or Paeoniflorin $(5 \mathrm{mg} / \mathrm{kg}$ and $10 \mathrm{mg} / \mathrm{kg})$. Data was analyzed by one-way ANOVA followedbytheScheffétest. $n=10 . * *<0.01$ vs. control group. mNSS, Modified neurological severity scores; MCAO, middle cerebral artery occlusion. 


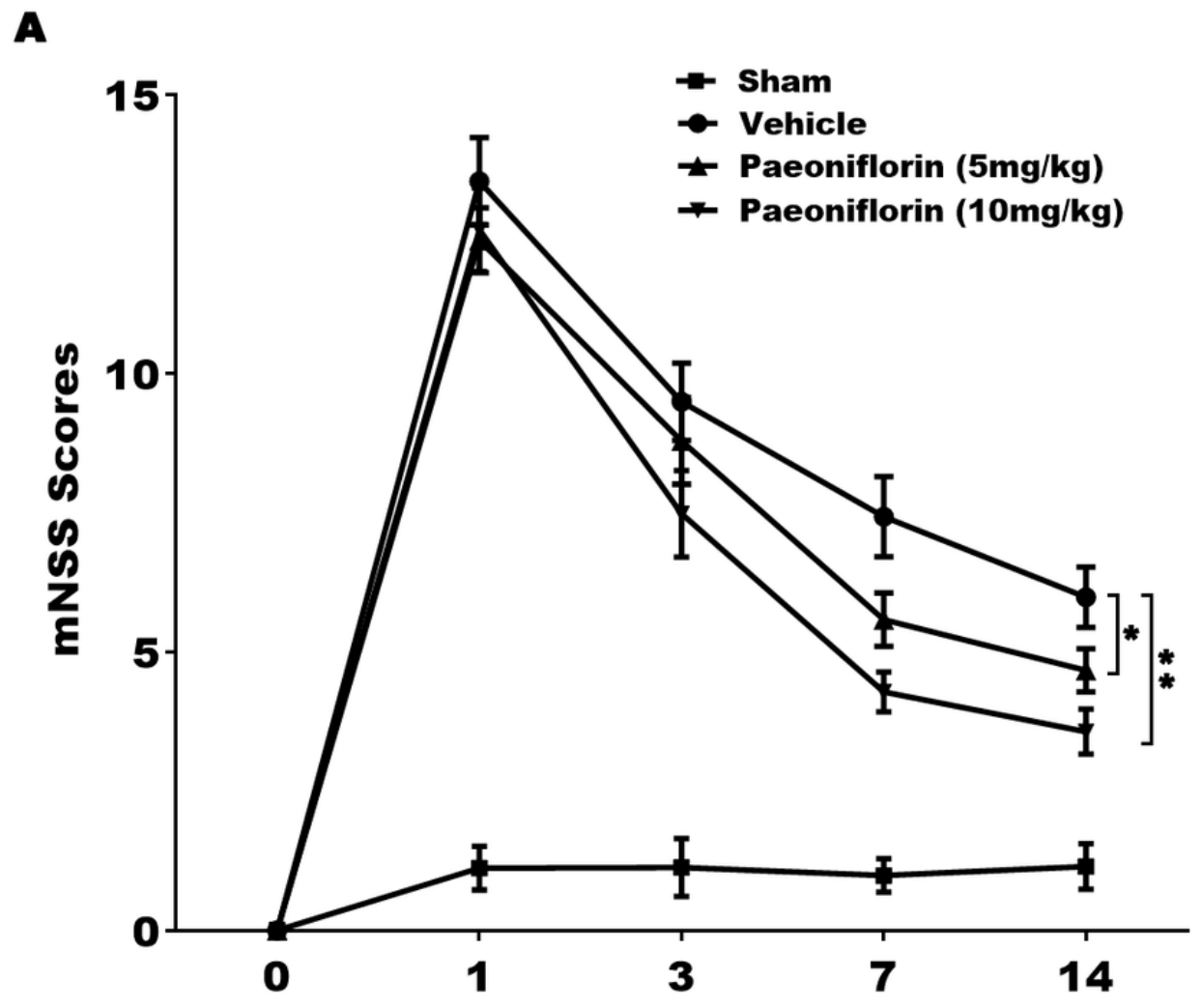

$\mathbf{B}$

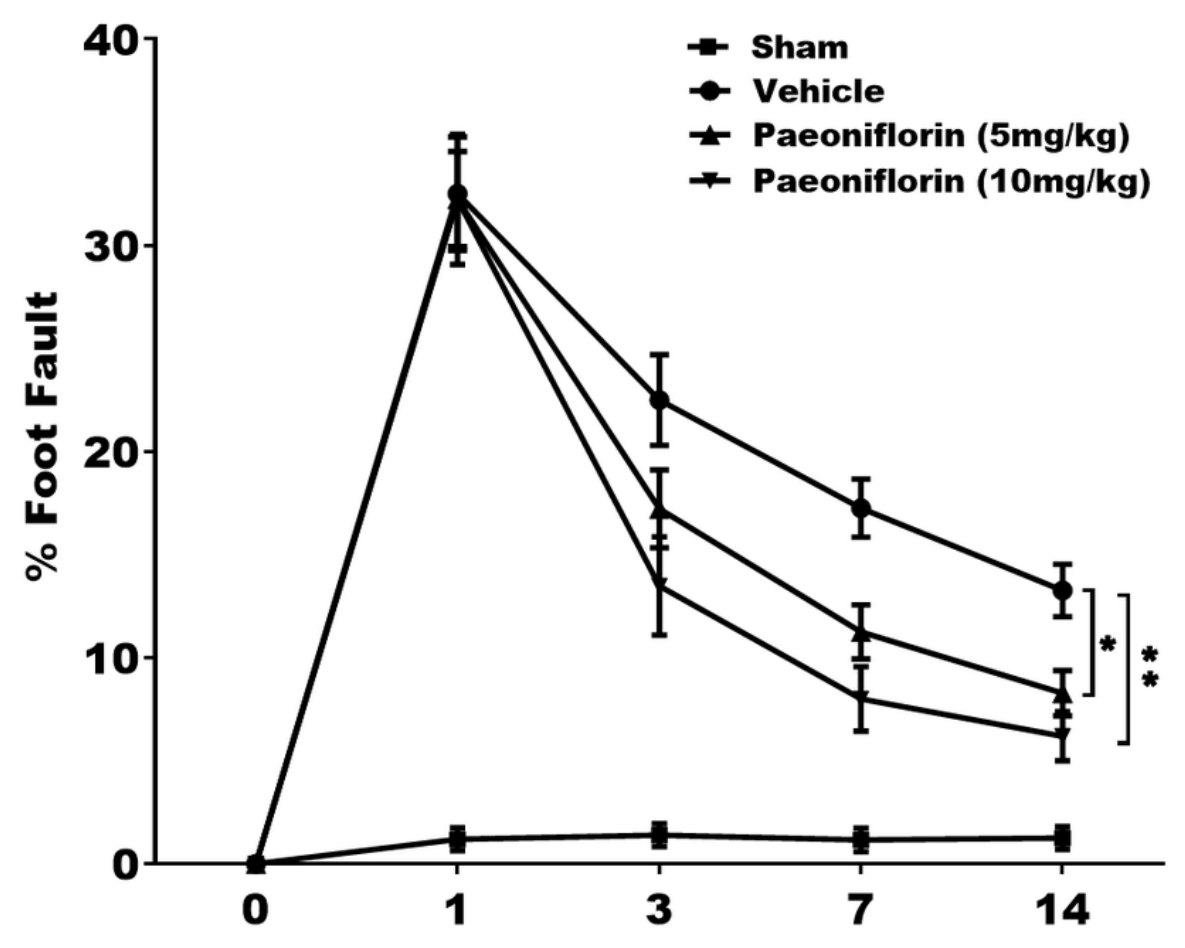




\section{Figure 2}

Paeoniflorin repressed microglia activation in rat stroke model

(A) The activation of microglia was analyzed using immunofluorescence analysis in ipsilateral cortex after MCAO and intraperitoneal injection of vehicle or Paeoniflorin $(10 \mathrm{mg} / \mathrm{kg}) \mathrm{n}=3$.

Scale bar, $20 \mu \mathrm{m}$ (B and C) Western blot and quantitative analysis of the protein expression level of Iba-1 in ipsilateral cortex after MCAO and intraperitoneal injection of vehicle or Paeoniflorin. $* P<0.05, * * P<0.01 . n=3$. MCAO, middle cerebral artery occlusion; Iba-1, lonized calcium bindingadaptor molecule-1. 
A
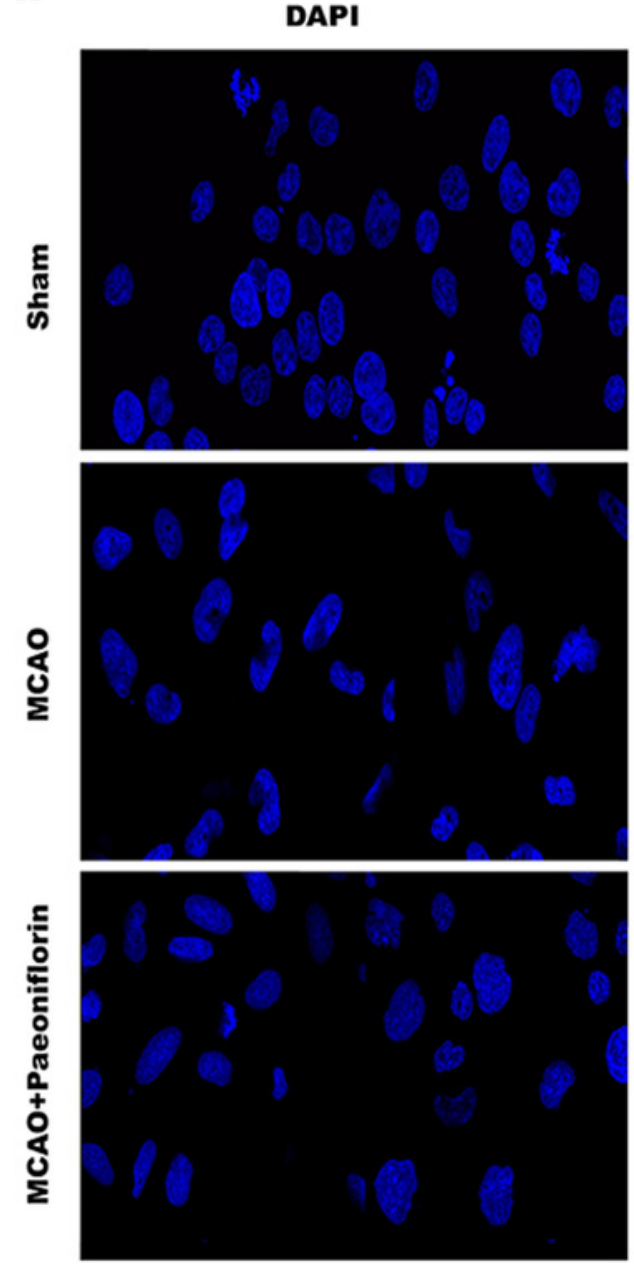

B
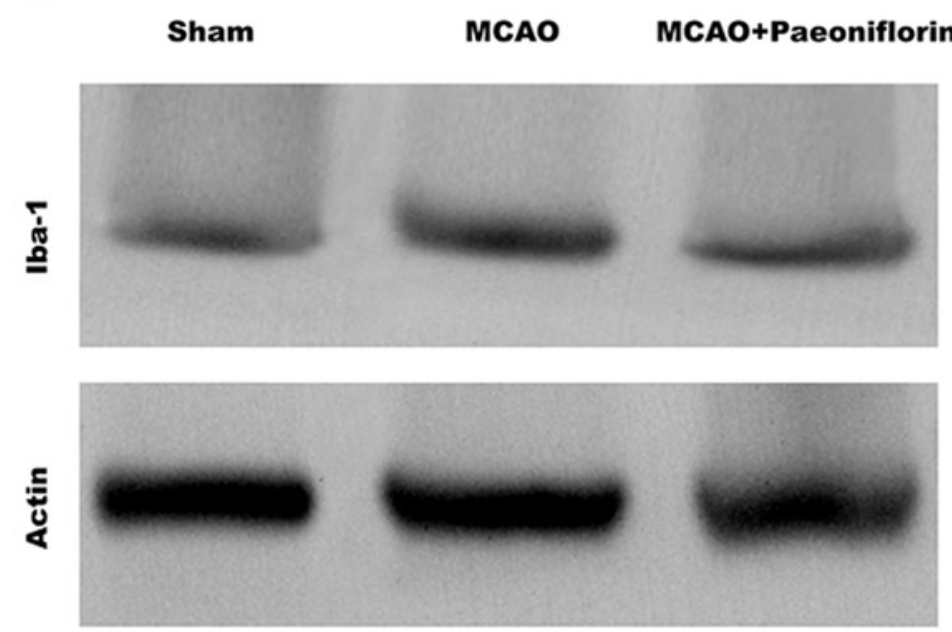

iba-1
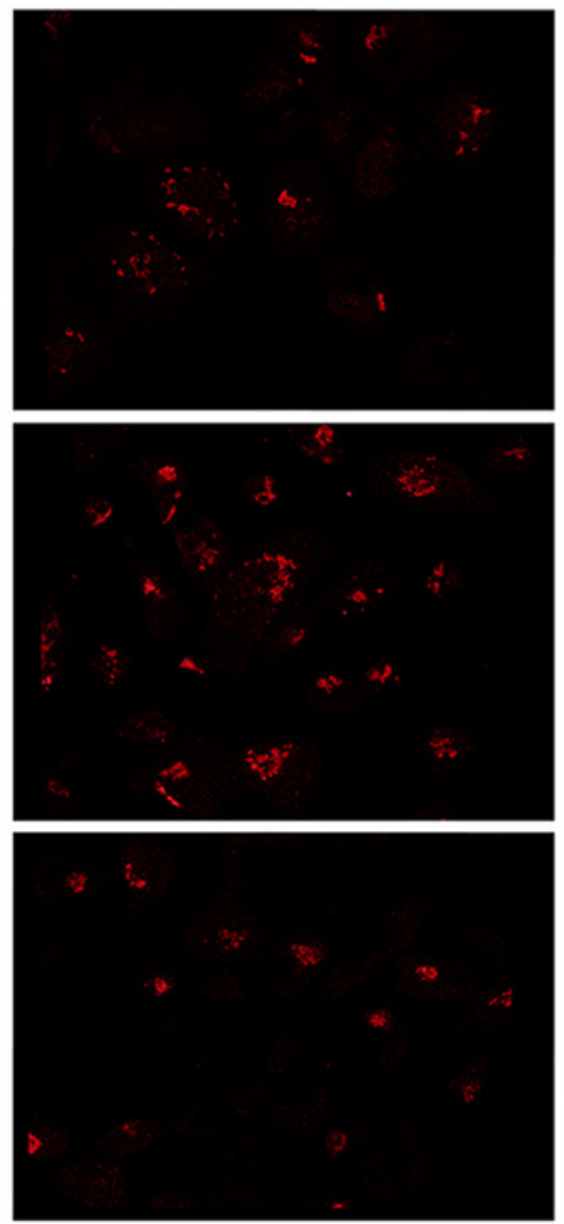

C

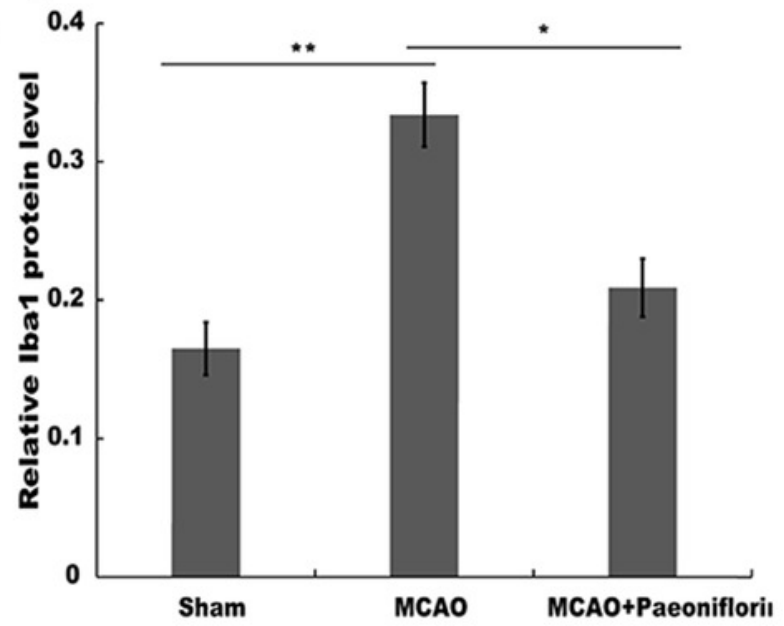




\section{Figure 3}

Paeoniflorin inhibits the secretion of pro-inflammatory cytokines in rat stroke model

(A-C) The levels of pro-inflammatory cytokines TNF-a (A), IL-1 $\beta$ (B) and IL-6 (C) were determined by ELISA assay using ELISA kits in brain tissues after MCAO and intraperitoneal injection of vehicle or Paeoniflorin $(10 \mathrm{mg} / \mathrm{kg}) . \mathrm{n}=3$. ${ }^{*} P<0.05,{ }^{*} * P<0.01$. IL-1 $\beta$, Interleukin-1 beta; TNF, tumor necrosis factor; IL-6, Interleukin-6; ELISA, enzyme linked immunosorbent assay; MCAO, middle cerebral artery occlusion.
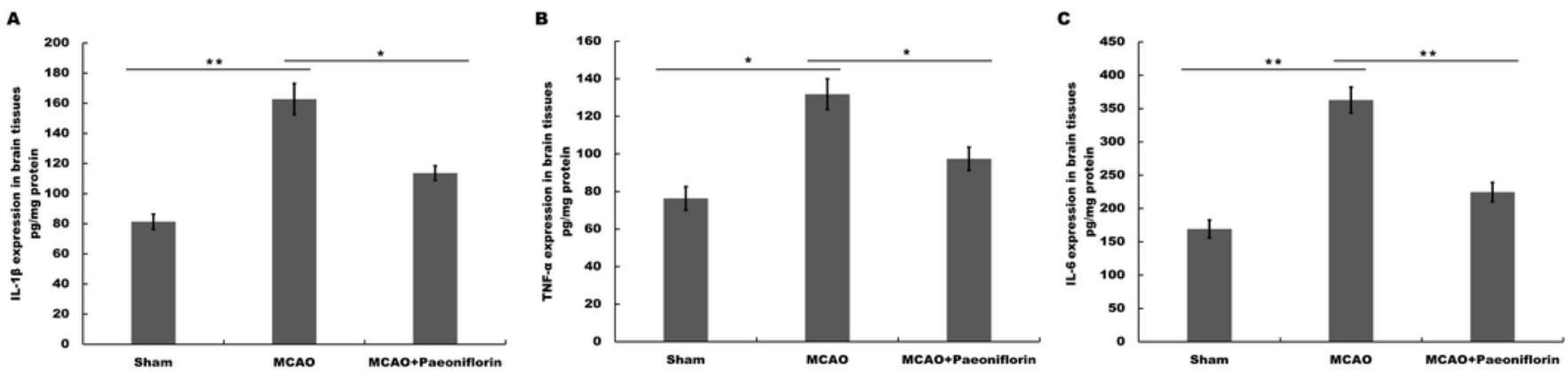


\section{Figure 4}

Paeoniflorin repressed microglial viability and inflammatory cytokines production in vitro

(A) BV2 microglia viability was determined by CCK-8 assay using CCK-8 kit in BV2 cells with

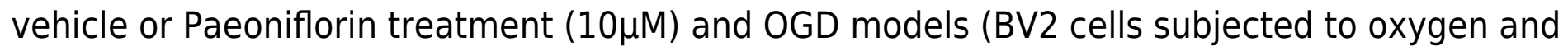

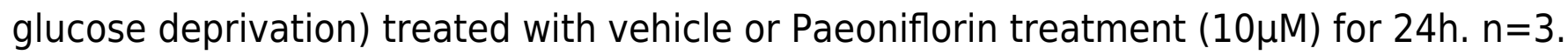
$* P<0$.05. (B-D) The mRNA expression levels of IL-1 $1 \beta$ (B), TNF- $\alpha$ (C), and IL-6 (D) were detected by qPCR analysis in BV2 cells with vehicle or Paeoniflorin treatment $(10 \mu \mathrm{M})$ and OGD models treated with vehicle or Paeoniflorin treatment $(10 \mu \mathrm{M})$ for $24 \mathrm{~h} . \mathrm{n}=3$. ${ }^{* * P}<0.01$. CCK-8, Cell Counting Kit-8; OGD, oxygen-glucose deprivation; IL-1 $\beta$, Interleukin-1 beta; TNF, tumor necrosis factor; IL-6, Interleukin-6; qPCR, Quantitative Real-time PCR. 

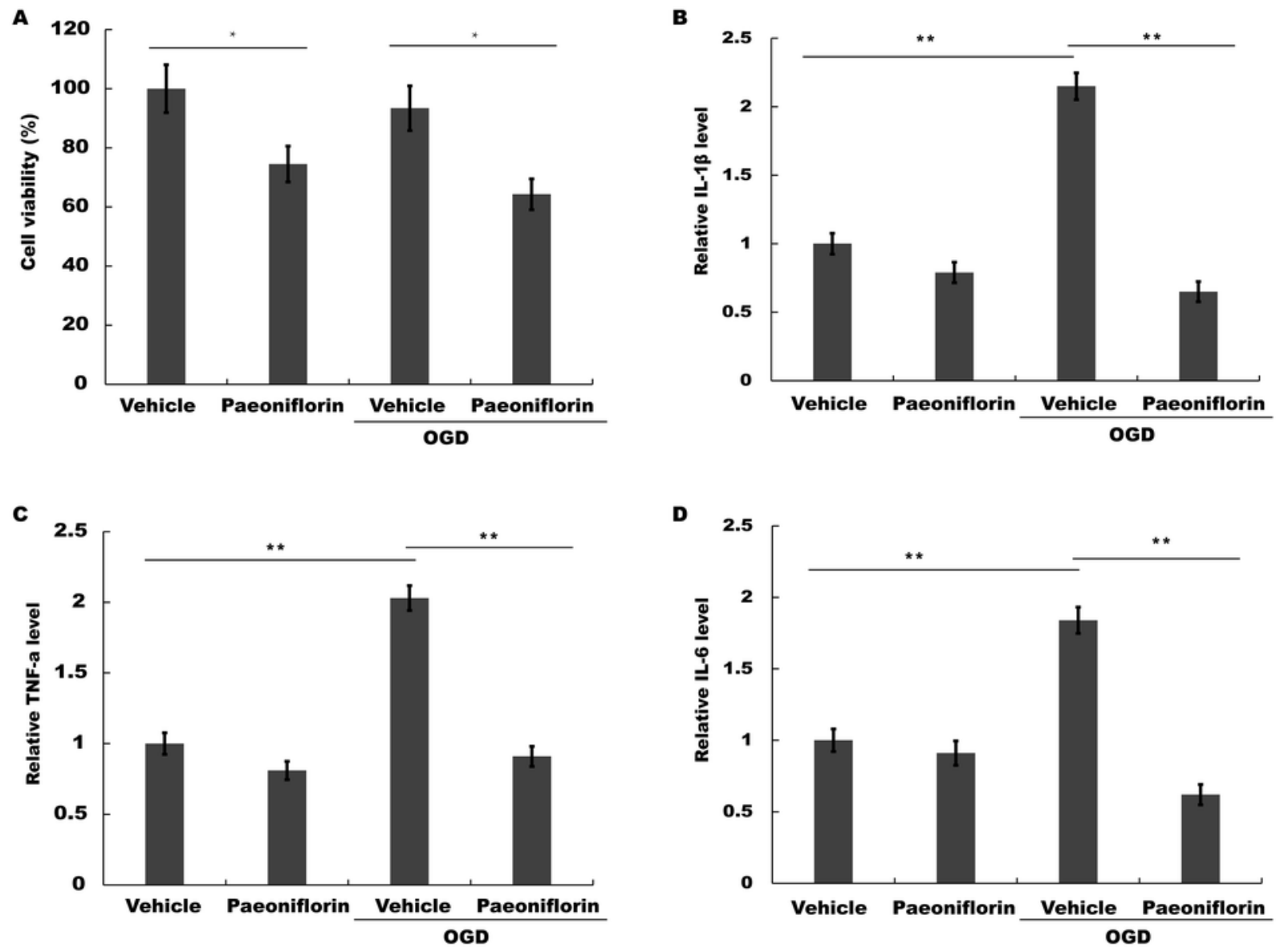


\section{Figure 5}

Paeoniflorin facilitated neurogenesis and vasculogenesis in rat stroke model

( $A$ and $B$ ) The marker of endothelial cells of cerebral blood vessels (VWF) expression was assessed in peri-infarct zone compared with control of rat stroke model after Paeoniflorin administration by Immunofluorescence. $n=3$. $* P<0.05$. ( $C$ and $D$ ) The indication of migrating neuroblasts (doublecortin) expression was assessed in peri-infarct zone compared with control of rat stroke model after Paeoniflorin administration by Immunofluorescence. ${ }^{*} P<<0.01$. ( $E$ and $F$ ) The effects of paeoniflorin on apoptotic cell was verified by TUNEL staining in rat stroke model treated with or without Paeoniflorin. Positive expression in TUNEL staining was exhibited. Rats treated with saline and PF (10 mg/kg) for 14 days after MCAO; $\mathrm{n}=5$. Scale bar, $50 \mu \mathrm{m}$. ${ }^{* * P}<0.01$. TUNEL, terminal dexynucleotidyl transferase (TdT)mediated dUTP nick end labeling. 
A

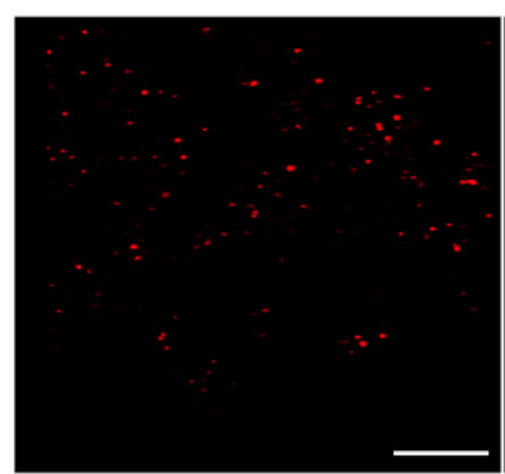

Control

c

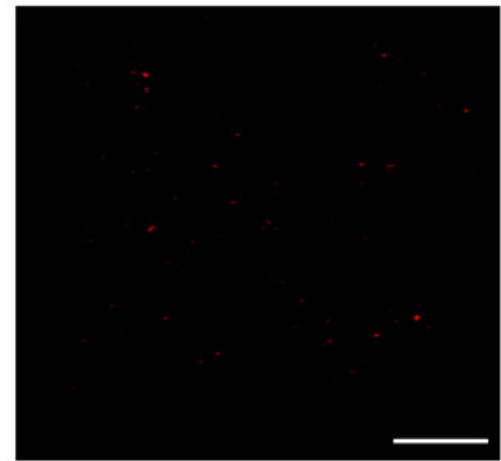

Control

E
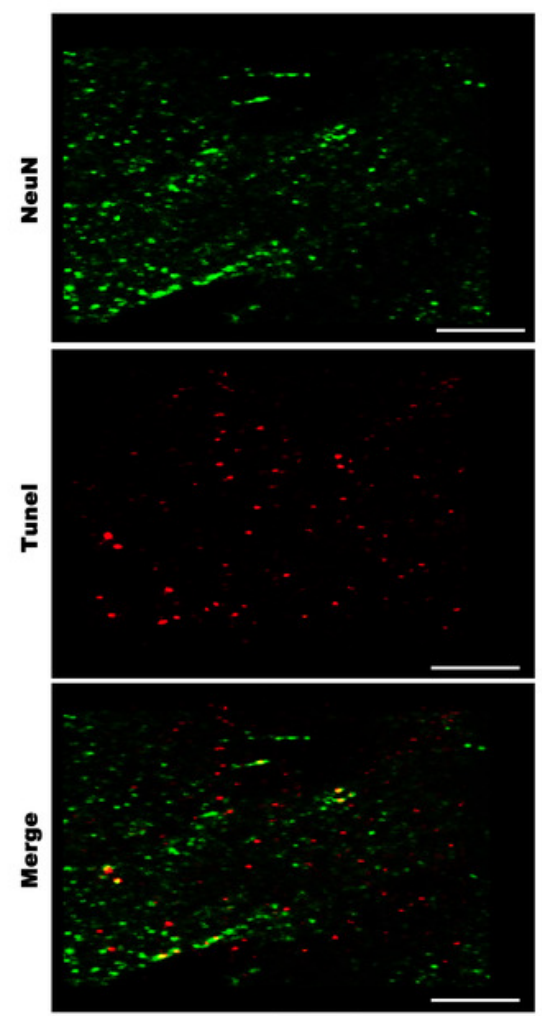

Control

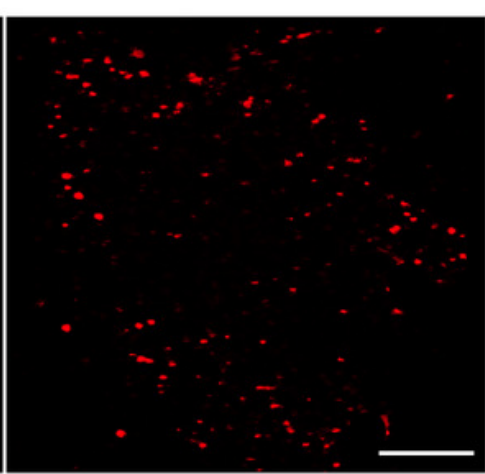

Paeoniflorin

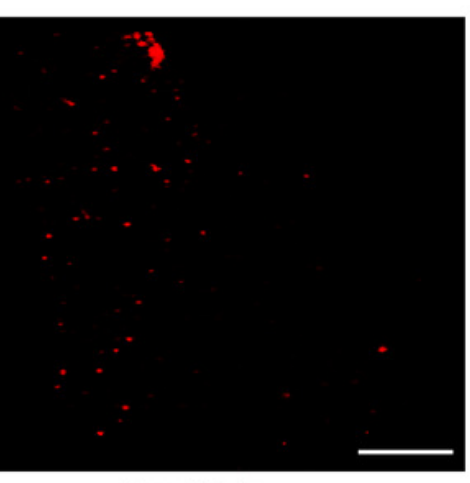

Paeoniflorin
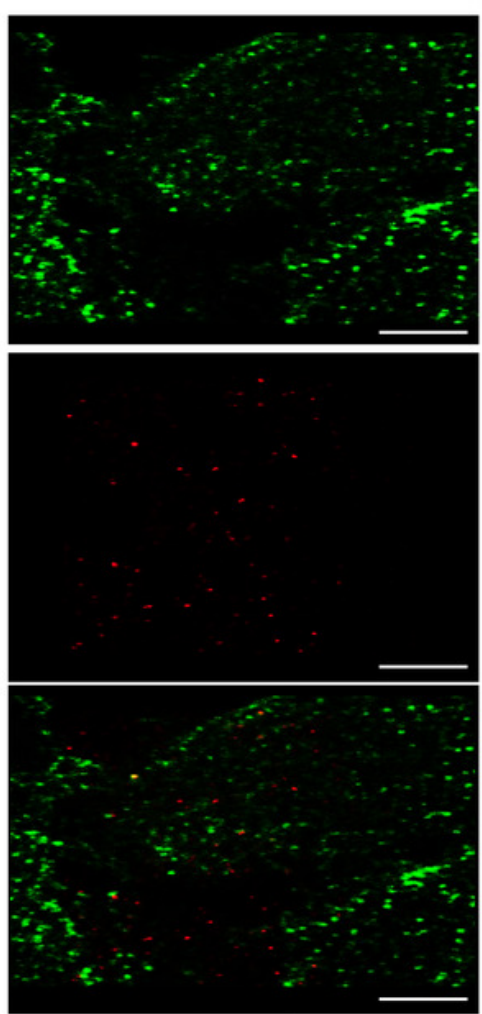

Paeoniflorin
B

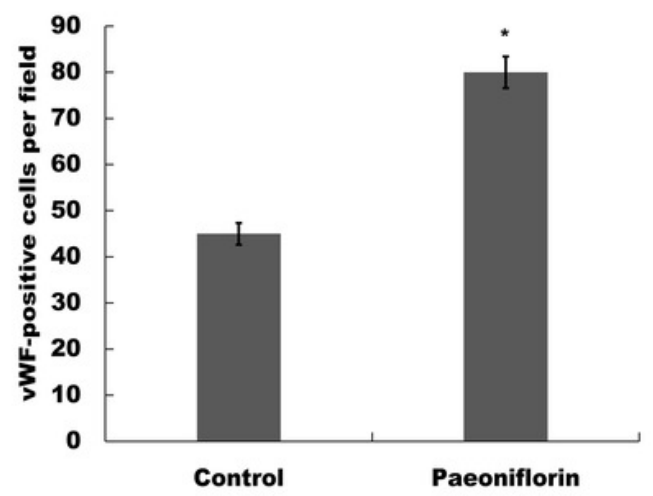

D

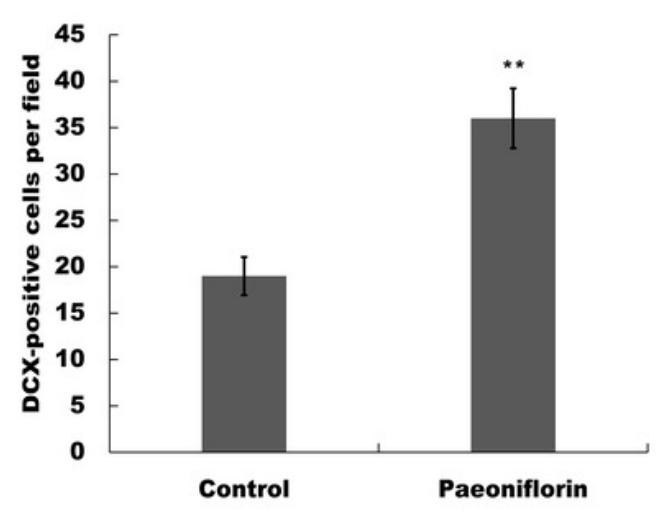

F

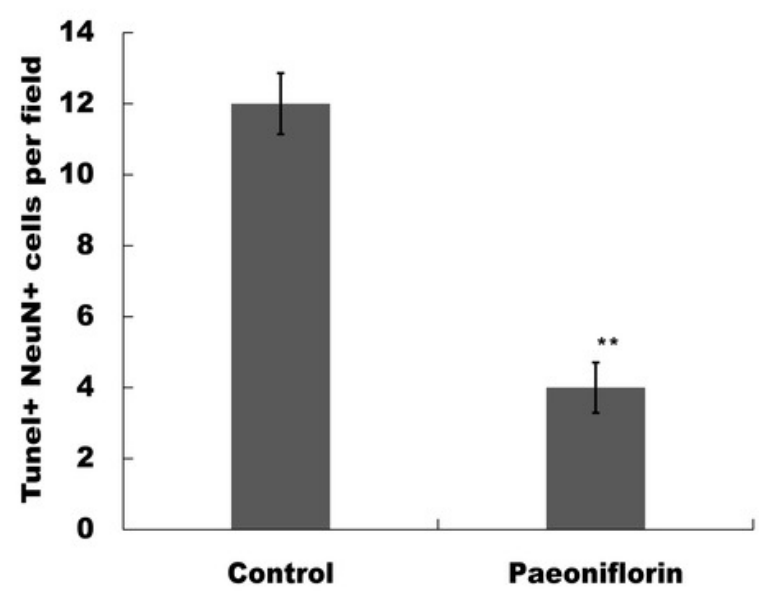

PeerJ reviewing PDF | (2020:07:50844:3:0:NEW 14 Jan 2021) 


\section{Figure 6}

Paeoniflorin repressed JNK and NK-KB signaling activation

( $A$ and $B$ ) Western blot and quantitative analysis of the protein expression level of JNK and phosphorylated JNK ( $p$-JNK) after MCAO and intraperitoneal injection of vehicle or Paeoniflorin. $\mathrm{n}=3$. ${ }^{* * P}<0.01, \# P<0.05$. ( $\mathrm{C}$ and $\mathrm{D}$ ) Western blot and quantitative analysis of the protein expression level of nuclear p65 and Histone $\mathrm{H} 3$ after MCAO and intraperitoneal injection of vehicle or Paeoniflorin. (E) Nuclear translocation of p65 was indicated using an immunofluorescence assay after MCAO and intraperitoneal injection of vehicle or Paeoniflorin. $\mathrm{n}=3 . * P<0.05$. Scale bar, $20 \mu \mathrm{m}$. MCAO, middle cerebral artery occlusion. 
A

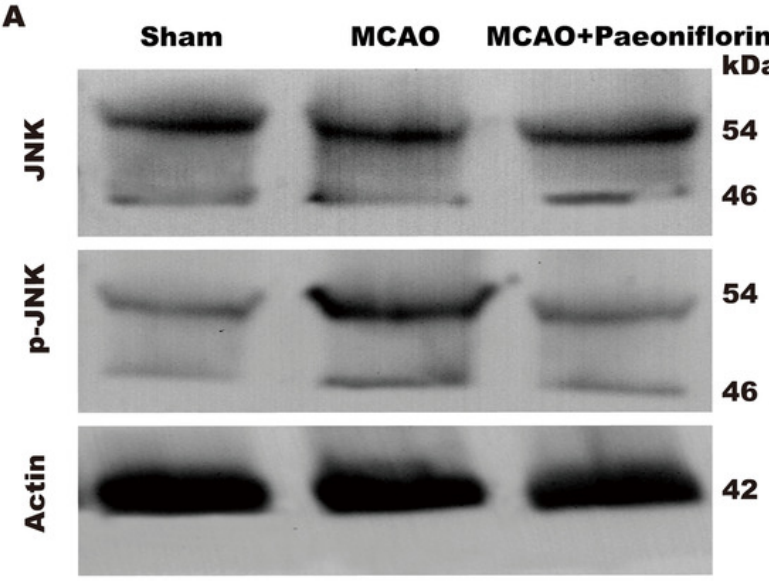

C

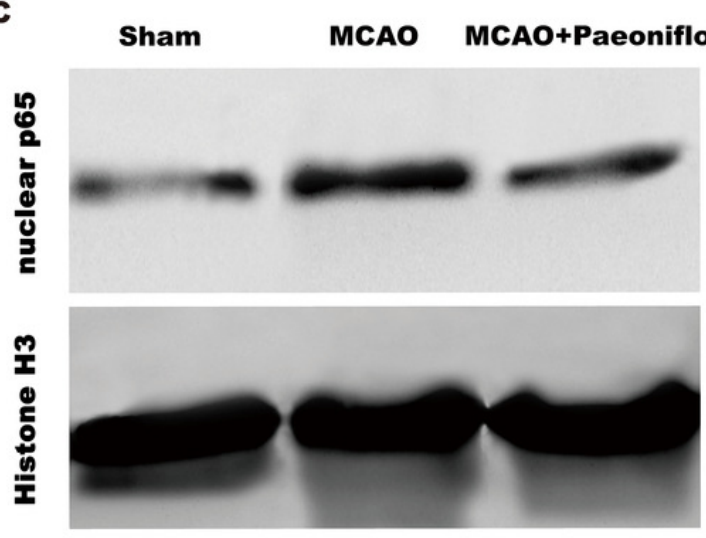

E
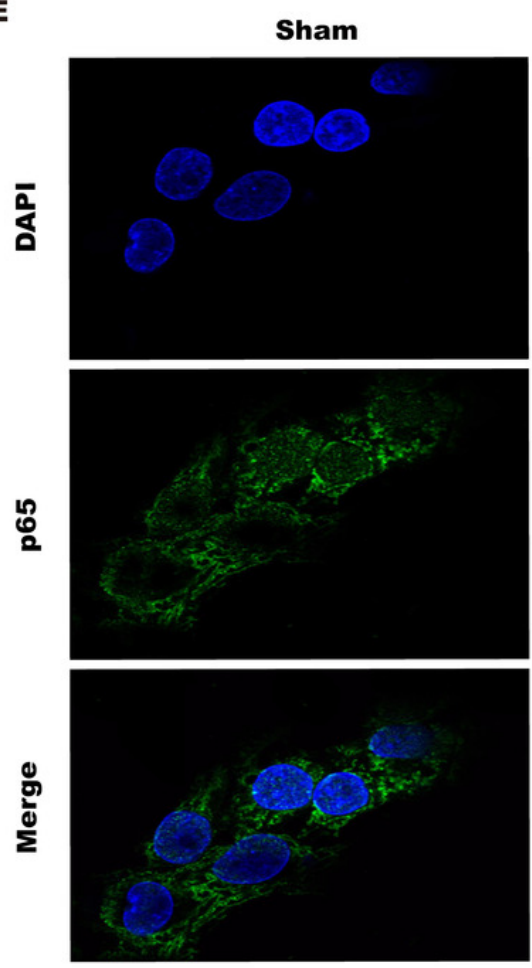
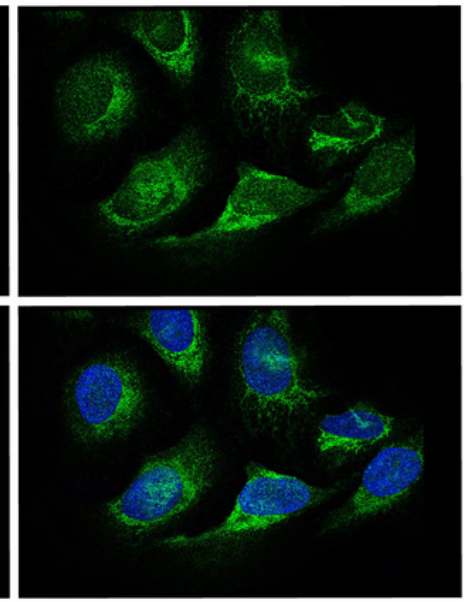

B

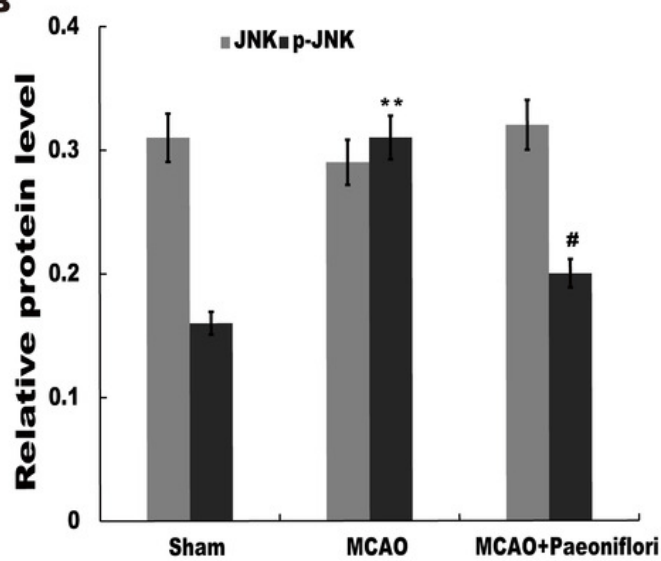

D

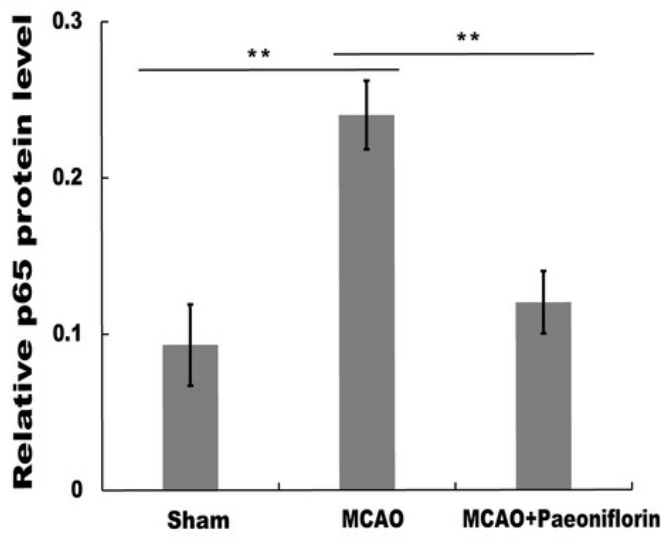

MCAO

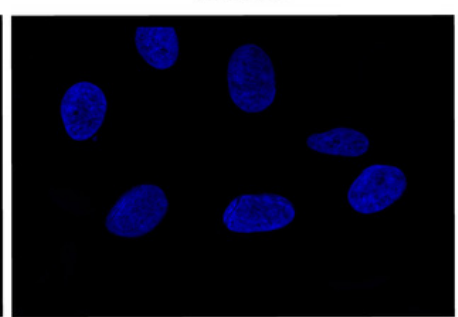

MCAO+Paeoniflorin
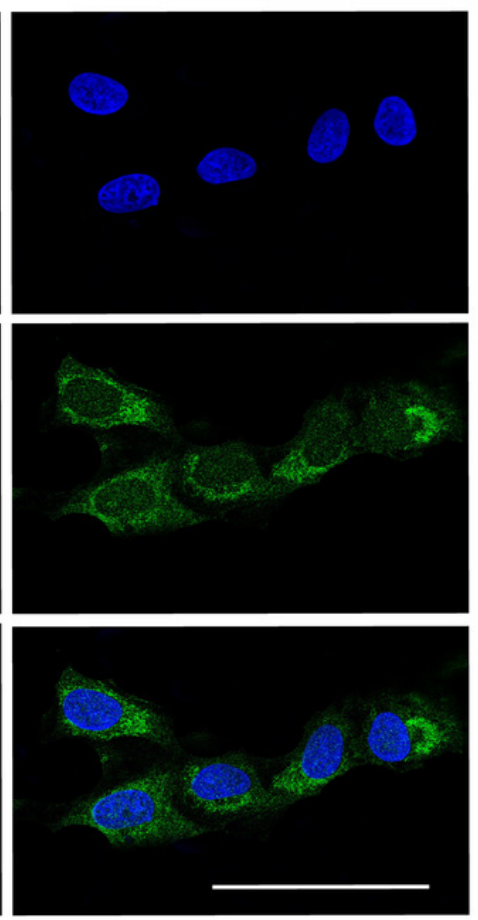